\title{
Qualidade de vida e autoestima de mulheres praticantes de musculação e ginástica em academia
}

\author{
Life quality and self-esteem of women muscular activity \\ and fitness practisers in the gym
}

Rômulo Tafarello, ${ }^{1}$ José Roberto Andrade do Nascimento Júnior, ${ }^{1}$ Daniel Vicentini de Oliveira 'Faculdade Metropolitana de Maringá (FAMMA), Maringá, PR, Brasil.

Recebido em: outubro 2015 / Aceito em: dezembro 2015

d.vicentini@hotmail.com

\section{RESUMO}

Objetivo: analisar a qualidade de vida (OV) e a autoestima $(A E)$ de mulheres praticantes de musculação e ginástica em academias do município de Maringá, estado do Paraná. Método: Foram avaliadas 30 mulheres, divididas em dois grupos de 15 pessoas, sendo grupo $A$ (praticantes de musculação) e o grupo B (praticantes de ginástica). Para avaliação de QV utilizou-se o questionário WHOQOF-BREF, e para avaliação da $A E$, a Escala de Autoestima desenvolvida por Rosenberg. Resultados: Não houve diferença significativa nos domínios de QV e na $A E$, quando comparados em função do tipo de exercício praticado pelas mulheres avaliadas, indicando que as mulheres praticantes de ginástica e musculação possuem percepções de QV e AE semelhantes. Conclusão: Verificou-se que são boas a boa a $Q V$ e a $A E$ das mulheres praticantes de musculação e ginástica do município de Maringá, estado do PR. Concluiu-se também que existe impacto significativo da autoestima na percepção da auto avaliação da QV nas mulheres praticantes de ginástica, e da $A E$ na percepção de $\mathrm{QV}$ nos domínios de relações sociais e meio ambiente nas praticantes de musculação.

Palavras-chave: Academia; Exercício físico; Promoção da saúde.

\section{ABSTRACT}

Objective: analyze the quality of life (OL) and self-esteem (SE) of women muscular activity and fitness practisers in the gyms of the city of Maringa, Parana. Method: 30 women divided into two groups of 15 people, with group A (muscular activity practisers) and group $B$ (gymnastics practisers). To $Q L$ assessment used the WHOQOF-BREF questionnaire, and for the SE evaluation, the Self-Esteem Scale developed by Rosenberg. Results: No significant differences in QL domains and SE compared regarding on the type of exercise practiced by women evaluated, indicating that women practitioners of gymnastics and weight training have similar QL and SE perceptions. Conclusion: It was found good QL and SE of women engaged in muscular activity and fitness in the city of Maringa, Parana, suggesting a positive impact of these modalities in the variables studied. It was also concluded that there is significant impact on SE in the perception of self-assessment of $O L$ in women engaged in gymnastics, and SE in the perception of $Q L$ in the fields of social relationships and environment in muscular activity practisers.

Keywords: Gym; Physical exercise; Health promotion.

\section{INTRODUÇ̃̃O}

A mulher é constantemente influenciada por fatores socioculturais que as conduzem a apresentar um conjunto de preocupações e insatisfações com seu corpo e com isso, diminuindo sua qualidade de vida (QV) e autoestima (AE). ${ }^{1}$ Cada vez mais essas mulheres têm buscado a prática de exercícios físicos, musculação ou ginástica em academias, visando melhorar sua QV e AE. Habitualmente, um dos locais mais escolhidos para cuidar do corpo e da mente são as salas de ginástica e as salas de musculação.

As academias de ginásticas se tornaram espaços onde se busca saberes, relacionados à postura, cuidados com o corpo, aprendizagem de exercícios físicos, além de ser um espaço privilegiado para a interação social. Nesse ambiente, as pessoas se sentem motivadas e reforçadas a praticarem os exercícios físicos como 
meio de aumentar a $A E$, uma vez que os frequentadores estão atrás de objetivos, sejam eles reais ou percebidos, ${ }^{2}$ como estética, saúde, aptidão física, disposição, atratividade e harmonia. ${ }^{3}$ Assim, o papel das academias é suprir a lacuna da falta de prática de regular exercício físico, com a vantagem de oferecer um serviço técnico especializado como forma de orientação.

O exercício físico tem sido muito valorizado, atualmente, por diversos fatores que interferem na QV, como o do estresse e questões ligadas ao sedentarismo, má alimentação, entre outros. Esses fatores fazem com que as pessoas busquem alternativas capazes de minimizar tais danos. ${ }^{4}$

Segundo a Organização Mundial de Saúde, QV é definida como a percepção do indivíduo de sua posição na vida, no contexto da cultura e do sistema de valores em que vive e em relação aos seus objetivos, expectativas, padrões e preocupações. ${ }^{5}$ Já, a AE constitui um sentimento de juízo, de apreciação, valorização, bem-estar e satisfação que o sujeito tem de si mesmo; encontra-se relacionada com alguns aspectos do cotidiano, tornando-se um aspecto central da saúde e do bem-estar psicológico. ${ }^{6}$

Matsudo e Matsudo ${ }^{7}$ reiteram a prescrição de exercício físico, enquanto fator de prevenção de doença e da QV e melhora da AE. Destaca-se que a saúde e a QV do homem podem ser preservadas e aprimoradas pela prática regular de atividade física e, principalmente, do exercício físico.

De acordo com Guedes, ${ }^{8}$ a musculação, uma modalidade de exercício físico, pode ser definida como um método de treinamento, cujo principal meio são os pesos (barras, anilhas, etc.) e a principal capacidade motora treinada é a força muscular. Para Monteiro ${ }^{9}$ a musculação vem sendo muito procurada por proporcionar um aprimoramento do condicionamento físico, da massa muscular, redução da massa gorda e melhora na QV. Já, a ginástica em academia, segundo Furtado ${ }^{10}$, consiste de aulas com diferentes objetivos e características, como a ginástica localizada, aulas em mini trampolim, step, aeróbica, dentre outras.

As melhorias proporcionadas pelo treinamento realizado na musculação nos aspectos neuromusculares (como o ganho de força), antropométricos (como redução do percentual de gordura) e metabólicos (como o aumento do metabolismo devido ao ganho de massa muscular) influenciam diretamente os aspectos psicológicos, como por exemplo, aumento da $A E$ e da motivação para a prática do exercício. Por outro lado, acredita-se que praticantes de ginástica em academia teriam altos escores de $\mathrm{QV}$, principalmente nos aspectos físico, social e psicológico. ${ }^{11}$

Posto isso, o presente estudo tem como objetivo analisar a $\mathrm{QV}$ e a $\mathrm{AE}$ de mulheres praticantes de musculação e ginástica nas academias do município de Maringá, estado do Paraná.

\section{MÉTODO}

O presente estudo caracteriza-se por ser quantitativo do tipo transversal, descritivo e analítico, aprovado pelo comitê de ética do Centro Universitário de Maringá (UNICESUMAR), pelo número do parecer 157.078, em

\section{3 de julho de 2015.}

A população do estudo foi composta de mulheres praticantes de musculação e ginástica em duas academias do município de Maringá, estado do Paraná. A amostra foi escolhida entre as mulheres que praticam musculação ou ginástica de academia, sendo de forma voluntária a aderência na pesquisa e sendo informadas quanto à justificativa, objetivos e procedimentos a serem realizados, de acordo com a resolução $n^{\circ}$ 196/96 do Conselho Nacional de Saúde. Após esses procedimentos, as participantes assinaram o Termo de Consentimento Livre e Esclarecido (TCLE).

Foram incluídas no estudo, mulheres com idade igual ou superior a 18 anos, até no máximo 60 anos, praticantes de musculação ou ginástica há pelo menos três meses e com frequência mínima de duas vezes por semana. Foram excluídas as mulheres praticantes de mais de uma das modalidades de exercício físico estudadas. A amostra final foi composta de 30 mulheres, divididas em dois grupos de 15 pessoas, sendo grupo A (praticantes de musculação) e o grupo B (praticantes de ginástica).

Para a caracterização das mulheres, foi utilizado um questionário, composto por informações referentes à idade, estado civil, situação ocupacional, frequência semanal de tempo de prática da musculação ou ginástica, e há quanto tempo praticam as mesmas.

A avaliação da QV foi realizada por meio do questionário WHOQOL-BREF, que é uma versão abreviada do WHOQOL-100, composto por 26 questões, das quais duas referem-se à percepção individual da $\mathrm{QV}$ e da percepção de saúde e as demais (24) são subdivididas em quatro domínios: físico, psicológico, relações sociais e meio ambiente. Cada domínio pode alcançar escores de 4 a 20 , sendo que quanto mais próximo de 20 , melhor a QV do indivíduo, no domínio avaliado. Somando-se os escores dos quatro domínios e das duas questões referentes à percepção do indivíduo, pode-se chegar a escores mínimos de 20 e máximo de 100. Quanto mais próximo de 100, melhor a QV global do avaliado. ${ }^{12}$

Quanto à avaliação da $A E$, o instrumento utilizado foi a Escala de Autoestima desenvolvida por Rosenberg..$^{13}$ Esta é uma medida unidimensional constituída por dez afirmações relacionadas a um conjunto de sentimentos de $A E$ e auto aceitação que avalia a autoestima global. Os itens são respondidos em uma escala tipo Likert de quatro pontos, variando entre concordo totalmente, concordo, discordo e discordo totalmente. Neste estudo, foi utilizada a versão adaptada para o português por Hutz ${ }^{14}$ cujos resultados iniciais já indicam a unidimensionalidade do instrumento e características psicométricas equivalentes às encontradas no arquivo original. ${ }^{13} \mathrm{O}$ escore obtido com a escala pode variar de 10 a 40, sendo calculado, somando-se as pontuações obtidas através das respostas dadas às 10 frases. Cada frase pode receber uma pontuação de, no mínimo, 1 e no máximo 4. Uma $A E$ satisfatória é definida como escore maior ou igual a 30 na Escala de Rosenberg e insatisfatória com escore menor que $30 .{ }^{15}$ Foi optado pela entrevista direta na aplicação de todos os instrumentos, depois da prática da modalidade, em horários pré-agendados pelo pesquisador.

Para a análise dos dados, foi utilizada frequência e percentual para as variáveis categóricas. Para as variáveis numéricas, inicialmente foi verificada a normalidade dos dados por meio do teste Shapiro-Wilk. Como os dados 
não apresentaram distribuição normal, foram utilizadas Mediana (Md) e Quartis (Q1; Q3) para a caracterização dos resultados. Como se pretendia verificar as maiores percepções em relação aos domínios de qualidade de vida dos sujeitos de uma forma geral, foi utilizada a ANOVA de medidas repetidas, após a verificação da esfericidade dos dados, seguida do Post Hoc de Bonferroni corrigido $(p<0,01)$. Na comparação entre os grupos (ginástica e musculação), foi utilizado o teste " $U$ " de Mann-Whitney. A correlação entre as variáveis ( $\mathrm{QV}$, $A E$, tempo de prática e frequência semanal) foi feita por meio da correlação de Spearman, sendo adotada a significância de $\mathrm{p}<0,05$.

Para verificar o impacto da $A E$ sobre os domínios de QV das mulheres praticantes de exercícios foi conduzido um modelo de regressão com as variáveis que obtiveram correlação acima de 0,40 (moderada). Apesar de a amostra ser considerada pequena para modelos de regressão múltipla, Knofczynski e Mundfrom ${ }^{16}$ apontam que o mínimo de dois sujeitos é aceitável para um modelo de regressão com bom nível de predição. A existência de outliers foi avaliada pela distância quadrada de Mahalanobis $\left(D^{2}\right)$ e a normalidade univariada das variáveis foi avaliada pelos coeficientes de assimetria $(|S k|<3)$ e curtose $(|K u|<10)$ uni e multivariada. Como os dados não apresentaram distribuição normal, utilizou-se a técnica de Bootstrap de Bollen-Stine para corrigir o valor dos coeficientes estimados pelo método da Máxima Verossimilhança implementado no software AMOS versão 18.0. Não foram observados valores de $D M^{2}$ indicadores da existência de outliers, nem correlações suficientemente fortes entre as variáveis que indicassem problemas com a multicolinearidade (Variance Inflation Factors <5,0). A interpretação dos coeficientes de regressão teve como referência: pouco efeito para coeficientes $<0,20$, médio efeito para coeficientes até 0,49 e forte efeito para coeficientes $>0,50(p<0,05)$.

\section{RESULTADOS E DISCUSSÃO}

Na tabela 1 são apresentados a frequência do perfil de mulheres do Grupo A (musculação) e Grupo B (ginástica) das academias da cidade de Maringá-PR.

Observa-se maior frequência de mulheres não casadas tanto no grupo B (66\%) quanto no grupo $A$ (80\%). A maioria das mulheres do Grupo A apresentavam até 30 anos de idade (66\%), enquanto no Grupo B a maior prevalência foi de mulheres até 40 anos $(46 \%$ de mulheres até 30 anos e $46 \%$ entre 31 e 40 anos).

No estudo realizado por Oliveira Junior ${ }^{17}$ em Criciúma, Santa Catarina, observa-se que a maior proporção de usuários das academias está entre 18 a 30 anos, representando $67 \%$ dos pesquisados. Saba ${ }^{18}$ também comenta que é comum que a média de idade entre frequentadores de academia seja baixa, em torno de 26 anos. No entanto, observou-se, no presente estudo, que a amostra pesquisada se encontra na faixa etária de 18 a 57 anos, demonstrando que o público pesquisado é amplo, o que contribui para encontrar pessoas de diferentes idades numa aula de ginástica ou num treino de musculação.

Os dados coletados e analisados mostraram que as mulheres pesquisadas são, na maioria, de alta escolaridade. Sabe-se que indivíduos com grau de instrução elevado apresentam uma maior conscientização da importância da prática de exercícios físicos, consequentemente. ${ }^{19}$

Quanto à renda mensal, em ambos os grupos pesquisados, a maioria concentra-se acima de dois salários mínimos. A independência financeira é um relevante elemento de positivação por parte das mulheres. Com relação à $\mathrm{QV}$, não houve diferença significativa $(p=0,285)$ entre as mulheres pesquisadas. O grupo A apresentou escore de 80,41 e o grupo B 74,58. A Tabela 2 apresenta a comparação dos domínios de QV predominantes das mulheres praticantes de exercício,

Tabela 1 - Distribuição de frequência do perfil de mulheres praticantes de musculação e ginástica em academias da cidade de Maringá-PR.

\begin{tabular}{|c|c|c|c|c|}
\hline \multirow[t]{2}{*}{ VARIÁVEIS } & \multicolumn{2}{|c|}{ Ginástica } & \multicolumn{2}{|c|}{ Musculação } \\
\hline & $f$ & $\%$ & $f$ & $\%$ \\
\hline \multicolumn{5}{|l|}{ Estado civil } \\
\hline Casada & 5 & 33,3 & 3 & 20 \\
\hline Não Casada & 10 & 66,7 & 12 & 80 \\
\hline Total & 15 & 100 & 15 & 100 \\
\hline \multicolumn{5}{|l|}{ Situação ocupacional } \\
\hline Ativo & 14 & 93,3 & 12 & 80 \\
\hline Inativo & 1 & 6,7 & 3 & 20 \\
\hline Total & 15 & 100 & 15 & 100 \\
\hline \multicolumn{5}{|l|}{ Renda mensal } \\
\hline Mais de 2 salários mínimos & 11 & 73,3 & 9 & 60 \\
\hline Menos de 2 salários mínimos & 4 & 26,7 & 6 & 40 \\
\hline Total & 15 & 100 & 15 & 100 \\
\hline \multicolumn{5}{|l|}{ Escolaridade } \\
\hline Superior completo & 10 & 66,7 & 6 & 40 \\
\hline Fundamental Completo & 5 & 33,3 & 9 & 60 \\
\hline Total & 15 & 100 & 15 & 100 \\
\hline \multicolumn{5}{|l|}{ Faixa Etária } \\
\hline Até 30 anos & 7 & 46,7 & 10 & 66,7 \\
\hline 31 a 40 anos & 7 & 46,7 & 2 & 13,3 \\
\hline 41 a 50 anos & 1 & 6,6 & 1 & 6,7 \\
\hline Mais de 50 anos & 0 & 0 & 2 & 13,3 \\
\hline Total & 15 & 100 & 15 & 100 \\
\hline
\end{tabular}


sem separá-las entre grupos.

Conforme se depreende da Tabela 2, os domínios de QV que mais prevaleceram entre as mulheres foram nas relações sociais ( $\mathrm{Md}=16,00)$ e físico $(\mathrm{Md}=$ $15,71)$, visto que apresentaram diferença significativa ( $p<0,01$ ) com o domínio que apresentou menor valor o meio ambiente $(\mathrm{Md}=14,50)$.

Tabela 2 - Comparação dos domínios de QV predominantes das mulheres praticantes de exercício.

\begin{tabular}{lc}
\hline Domínios de Qualidade de Vida & Md $(\mathbf{0 1} ; \mathbf{0 3})$ \\
\hline Domínio 1 - Físico & $15,71(14,71 ; 17,28)^{\mathrm{a}}$ \\
Domínio 2 - Psicológico & $15,33(14,00 ; 16,00)$ \\
Domínio 3 - Relações sociais & $16,00(14,67 ; 17,33)^{\mathrm{b}}$ \\
Domínio 4 - Meio ambiente & $14,50(13,50 ; 16,00)^{\mathrm{a}, \mathrm{b}}$ \\
Domínio 5 - Auto avaliação & $16,00(14,00 ; 16,00)$ \\
\hline
\end{tabular}

*Diferença significativa: $p<0,01$ entre: a) Físico e Meio ambiente; b) Relações sociais e Meio ambiente.

$\mathrm{Na}$ prática da musculação, mesmo sendo de forma individual, o praticante tem contato com outras pessoas, e na ginástica a atividade é feita sempre em grupos, aumentando assim, o papel dos praticantes na sociedade e a ampliação do círculo de amizades, o que pode justificar a alta QV no domínio relações sociais.

Stahl $^{20}$ verificou que o ambiente social é forte indicador para ser fisicamente ativo. Tais resultados fortalecem as pesquisas que previamente constataram associação positiva entre apoio social e atividade física, numa variedade de amostras da população.

Em relação ao domínio físico, observou-se diferença significativa para as praticantes de exercício físico para ambos os grupos. Mazini Filho ${ }^{21}$ confirma que a prática regular de exercícios pode contribuir para melhor $\mathrm{QV}$, ao oferecer diversas vantagens como fortalecimento muscular; aumento da resistência óssea; adequação do perfil lipídico; aumento da sensibilidade à insulina; minimização das modificações teciduais ocasionadas pelo envelhecimento; melhora no funcionamento hemodinâmico do coração; controle da hipertensão e melhora da imunidade, ocasionando menor incidência de infecções.

Tahara, Schwarts e Silva ${ }^{4}$ descrevem que a questão estética é hoje uma preocupação de grande parcela da população, bem como adotar para si um estilo ou hábito saudável e, por conseguinte, melhorar a QV.

O único domínio em que as mulheres obtiveram menor escore foi o meio ambiente, o que compreende aspectos relacionados à segurança, recursos financeiros, informações, transporte e lazer que variam de acordo com a região. Uma boa situação socioeconômica mostra-se associada a uma melhor exigência a instalações e ao ambiente em si das academias. Geralmente, como verificado nas academias pesquisadas, o calor nas academias é grande, mesmo com os climatizadores e ventiladores ligados. Como o aluno permanece neste ambiente, durante um tempo substancialmente superior a 45 minutos, isto afetaria negativamente sua percepção de QV, com relação a este domínio.

Atualmente, a tendência é aceitar que as características ambientais podem estar consistentemente associadas ao exercício físico, em várias abordagens. Os fatores como o clima, as facilidades de acesso às instalações influenciaram de forma negativa no presente estudo. A falta de ventilação, o calor excessivo, o ambiente fechado da sala de ginástica e de musculação favorecem, que o domínio meio ambiente, tenha o menor escore.

Também corroborando com nossos resultados, um estudo realizado em uma academia no Rio de Janeiro, com objetivo de descrever o índice de QV e o índice de condicionamento físico, sob o ponto de vista do fitness físico, de indivíduos praticantes de musculação e não-atletas, com uma amostra de 34 sujeitos com idade entre 20 e 60 anos em que $68 \%$ dos testados eram do sexo feminino e $32 \%$ do sexo masculino, demonstrou que a realização do exercício físico orientado com o intuito de melhorar o condicionamento físico, como a musculação, proporcionou uma boa percepção da qualidade de vida. Ao avaliar os domínios, constatou-se que o maior valor foi o social, enquanto o de menor valor foi o ambiental. ${ }^{22}$ Cabe lembrar que a QV depende das expectativas individuais e do meio no qual o indivíduo está inserido.

A análise da escala de $A E$ de ambos os grupos demonstrou que todos os valores obtidos na amostra estudada ficaram próximos ao escore máximo, ou seja, 40 , sugerindo que, de forma geral, a $A E$ foi percebida como boa, tanto nas mulheres do grupo $A$, quanto nas do grupo $B$.

Pode-se sugerir que o resultado encontrado no presente estudo esteja atribuído à participação em exercícios físicos, conforme já vem sendo apontado em outros estudos ou ainda a uma tendência de maximizar a $A E$, que parece ser um problema do instrumento utilizado que, mesmo com respaldo e importância internacional, identifica escores de AE elevados na maioria dos estudos em que é utilizado. ${ }^{23}$

Esses resultados corroboram com os encontrados na literatura quando Mazo, Cardoso e Aguiar $^{24}$ e Safons ${ }^{25}$ explicam que, em seus estudos, foi verificado um aumento de respostas positivas no final de um programa de exercício físico, concluindo que a participação no mesmo contribui de forma significativa, para a melhoria da $A E$ das pessoas. A prática de exercício físico aumenta a $A E$, a $Q V$, é promotora de saúde e, além disso, aumenta a produtividade de quem a pratica.

Não houve diferença significativa $(p>0,05)$ nos domínios de QV e na AE quando comparados em função do tipo de exercício praticado pelas mulheres avaliadas (Tabela 3), indicando que as mulheres praticantes de ginástica e musculação possuem percepções de QV e AE semelhantes. O treino das duas modalidades abrange todo o corpo e resulta numa tonificação muscular extremamente equilibrada e distribuída. Quanto ao nível mental, além de combater o stress, transmite ao praticante uma sensação de confiança, melhorando desta forma, a $A E$ e a $Q V$ da pessoa.

A musculação, nos dias atuais, já não consiste em apenas levantar peso, ao contrário, se transformou em importante meio para garantir benefícios que agregam melhorias significativas para a QV, sejam estes psicológicos, biológicos ou sociais. Estudos descritos por Mello ${ }^{26}$ verificaram que o exercício físico sistematizado pode acarretar diversos benefícios, tanto na esfera física, quanto mental do ser humano.

Considerando que, grande número de mulheres vive numa cultura competitiva, ambiente que pode fa- 
Tabela 3 - Comparação dos domínios de QV e AE de mulheres praticantes de musculação (Grupo A) e ginástica (Grupo B) da cidade de Maringá-PR.

\begin{tabular}{|c|c|c|}
\hline VARIÁVEIS & $\begin{array}{c}\text { Ginástica }(n=15) \\
\operatorname{Md}(01 ; 03)\end{array}$ & $\begin{array}{c}\text { Musculação (n = 15) } \\
\text { Md (Q1;03) }\end{array}$ \\
\hline Autoestima & $35,00(30,00 ; 36,00)$ & $33,00(28,00 ; 36,00)$ \\
\hline \multicolumn{3}{|l|}{ Qualidade de vida } \\
\hline Domínio 1 - Físico & $15,42(14,28 ; 16,57)$ & $16,00(15,42 ; 17,71)$ \\
\hline Domínio 2 - Psicológico & $15,33(14,00 ; 15,33)$ & $15,33(14,67 ; 16,67)$ \\
\hline Domínio 3 - Relações sociais & $14,67(13,33 ; 17,33)$ & $17,33(16,00 ; 17,33)$ \\
\hline Domínio 4 - Meio ambiente & $14,50(13,50 ; 16,00)$ & $14,50(13,00 ; 16,00)$ \\
\hline Domínio 5 - Auto avaliação & $16,00(14,00 ; 16,00)$ & $16,00(14,00 ; 16,00)$ \\
\hline
\end{tabular}

* Diferença significativa: $p<0,05$ - Teste "U" de Mann-Whitney.

vorecer o aparecimento de sentimentos de inadequação e baixa $A E^{27}$, torna-se de grande relevância o incentivo a novos estudos enfocando AE em mulheres, que possam servir como referência aos pesquisadores na comparação de seus resultados, assim como fornecer subsídios ao sistema de Saúde Pública, no estabelecimento de novas intervenções na promoção de QV da população.

A Tabela 4 apresenta a correlação dos domínios de QV com AE. Nas mulheres do Grupo B verificou-se correlação significativa $(p<0,05)$, positiva e moderada $(r>0,40)$ da $A E$ com o Domínio de Auto avaliação $(r=0,53)$. Assim sendo, observa-se melhor AE quando o domínio de auto avaliação é melhor, ou vice-versa.

Tabela 4 - Comparação dos domínios de QV predominantes das mulheres praticantes de exercício.

\begin{tabular}{lc}
\hline Variáveis & Autoestima \\
\hline Praticantes de ginástica & \\
Domínio 1 - Físico & 0,05 \\
Domínio 2 - Psicológico & 0,48 \\
Domínio 3 - Relações sociais & 0,37 \\
Domínio 4 - Meio ambiente & 0,37 \\
Domínio 5 - Auto avaliação & $0,53^{*}$ \\
Praticantes de Musculação & \\
Domínio 1 - Físico & 0,37 \\
Domínio 2 - Psicológico & $0,61^{*}$ \\
Domínio 3 - Relações sociais & $0,68 *$ \\
Domínio 4 - Meio ambiente & $0,74 *$ \\
Domínio 5 - Auto avaliação & 0,44 \\
\hline
\end{tabular}

Coeficiente de correlação de Sperarman - ${ }^{*} p<0,05$.

Para verificar o impacto da $A E$ nos domínios de QV das mulheres de ambos os grupos, após a análise da correlação, foi conduzido um modelo de regressão entre os domínios de QV e AE que apresentaram correlação moderada. Nas mulheres do Grupo B, verificou-se que (Figura 1) o Domínio de Auto avaliação apresentou impacto significativo $(p<0,05)$ na variabilidade da $A E$ (50\%) (Figura 1).

As mulheres que participavam do grupo de ginástica tiveram sentimentos positivos em relação ao próprio corpo e níveis mais altos de $A E$ gerada dentro do grupo. ${ }^{28}$ A AE tem sido desenvolvida positivamente com a intervenção de programas de exercícios físicos e esportes, tendo resultados na QV e bem-estar mental, e que esta variável está correlacionada diretamente à autoimagem e auto avaliação. ${ }^{29}$

Já, nas mulheres do grupo $A$, observou-se correlação significativa $(p<0,05)$, positiva e moderada
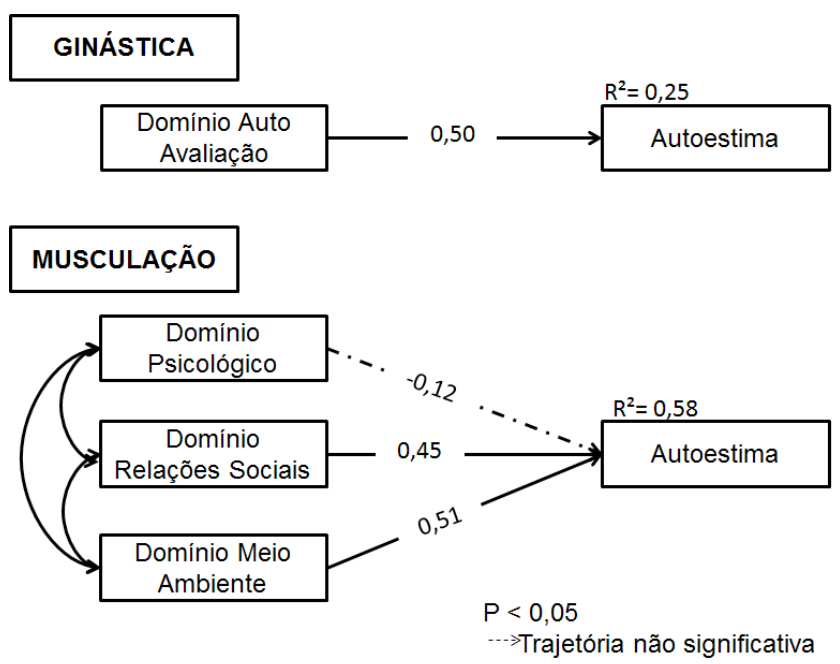

Figura 1 - Modelo de regressão do impacto da AE sobre a QV das mulheres praticantes de exercícios.

$(r>0,40)$ da Autoestima com os Domínios Psicológico $(r=0,61)$, Relações Sociais $(r=0,68)$ e Meio Ambiente $(r=0,74)$ (Tabela 4). Em relação às trajetórias individuais do modelo de regressão (Figura 1), verificou-se que o aumento das percepções das mulheres do grupo A nos Domínios Relações Sociais e Meio Ambiente possui um efeito significativo $(p<0,05)$ forte $(ß>0,50)$ sobre a $A E$ $(0,58)$. Já, a trajetória do Domínio Psicológico para a $A E$ não apresentou impacto significativo $(p>0,05)$. Estes resultados sugerem que a prática de musculação não apenas favorece uma melhoria/manutenção da QV e da AE de seus praticantes, como potencializa os benefícios advindos de um estilo de vida mais ativo, nos aspectos sociais e ambientais.

A prática de musculação estimula a socialização, proporciona maior empenho na busca de objetivos e melhora a AE. Resultado este, que se observou na pesquisa do autor acima, pois $94 \%$ dos adolescentes relacionaram a melhora da $\mathrm{AE}$ com a prática da musculação. ${ }^{30}$

Esses dados encontrados se assemelharam com os de Coelho-Ravagnani, ${ }^{31}$ em estudo sobre o efeito de programa de musculação para mudança do estilo de vida sobre a percepção de saúde em adultos, demonstrando que $96 \%$ dos entrevistados mencionaram mudanças relacionadas à dimensão física, 67\% nas psicológicas e 83\% em dimensão social.

As relações sociais são um dos atrativos da academia, apesar de não ser um espaço construído com esse enfoque; promove experiências de lazer a partir do 
interesse social. Confirma-se que esses elementos da vida humana devem caminhar juntos e não isoladamente, contribuindo para o bem-estar das pessoas.

O domínio psicológico para a $\mathrm{AE}$ não apresentou impacto significativo. Ressalta-se que o domínio psicológico apresenta a faceta espiritualidade, religião e crenças pessoais, buscando avaliar o sentido que o indivíduo encontra na vida. Este domínio considera os sentimentos positivos e negativos, a $A E$, a espiritualidade, a imagem corporal e o pensar e aprender. Assim, a $A E$ parece associar-se mais a uma medida de bem-estar subjetivo (satisfação com a vida) do que psicológico, o que pode estar relacionado com a própria definição. A $A E$, como avaliação da vida no seu todo, parece ter em comum um componente valorativo e emocional: gostar de si ou da vida que tem. Esse componente emocional parece não estar presente nas medidas de bem-estar psicológico.

O estudo apresenta algumas limitações, como a ausência de um grupo controle constituído por mulheres não praticantes de exercícios físicos. Outro aspecto que deve ser destacado refere-se aos locais distintos de coleta dos dados, fato que pode resultar em diferentes mensurações e classificações. Entretanto, mesmo com as limitações apontadas, esta pesquisa poderá fornecer aos profissionais da saúde importantes conhecimentos sobre a importância de se incentivar e intensificar a prática dos exercícios físicos, como um dos determinantes da promoção de saúde da mulher, principalmente no que se refere aos aspectos relacionados a $\mathrm{QV}$ e $\mathrm{AE}$.

\section{CONSIDERACÕ̃ES FINAIS}

Verificou-se que é a boa a QV e a AE das mulheres praticantes de musculação e ginástica do município de Maringá, não apresentando diferenças significativas entre os grupos. Concluiu-se também que existe impacto significativo da autoestima na percepção da auto-avaliação da QV nas mulheres praticantes de ginástica, e da $A E$ na percepção de QV nos domínios de relações sociais e meio ambiente nas praticantes de musculação.

\section{REFERÊNCIAS}

1. Saba F. Mexa-se: atividade física, saúde e bem-estar. São Paulo: Takano, 2003.

2. Fernandes R. Significados da ginástica para mulheres praticantes em academia. Motriz 2005;11(2):97-102.

3. Vilela GF, Rombaldi AJ. Perfil dos frequentadores das academias de ginástica de um município do Rio Grande do Sul. Rev Bras Promoç Saúde, Fortaleza 2015;28(2):206-215. doi: 10.5020/18061230.2015.p206

4. Tahara AK, Silva KA. A prática de exercícios físicos na promoção de um estilo de vida ativo. Lecturas: Educacion Fisicay Deportes 2003;9(61):65-80.

5. Gonçalves A, Vilarta R. Qualidade de Vida: identidades e indicadores. São Paulo: Manole; 2004.

6. Amatuzzi MM. Vivências de felicidade de pessoas idosas. Estud Psicol. 2008;25(2):303-07. doi: 10.1590/S0103 $166 \times 2008000200014$.

7. Matsudo SM, Matsudo VKR. Prescrição e benefícios da atividade física na terceira idade. Rev Bras Ciênc Mov 1992;6(4):19-30.

8. Guedes DP, Souza Júnior TP, Rocha AC, Treinamento personalizado em musculação. São Paulo: Icone 2008:456.

9. Monteiro AG. Treinamento personalizado. São Paulo: Phorte, 2000.

10. Furtado PR. Do Fitness ao Wellness: os três estágios do desenvolvimento nas academias de ginástica. Rev Pensar a Prática 2009;12(1):1-11.

11. Nahas MV. Atividade física, saúde e qualidade de vida. Londrina: Midiograf, 2001.

12. Fleck MPA, Chachamovich E. Desenvolvimento da versão em português do instrumento de avaliação de qualidade de vida da OMS (WHOQOL-100). Rev Bras Psiquiatria 1999; 21(1):19-28.

13. Rosenberg M. Conceiving the Self. New York: Basic Books. 1979.

14. Hutz CS. Adaptação da escala de autoestima de Rosenberg. Universidade Federal do Rio Grande do Sul. Porto Alegre-RS, 2000.

15. Simonetti VMM. Revisão crítica de algumas escalas psicossociais utilizadas no Brasil. Rio de Janeiro: Universidade Gama Filho, 1989.

16. Knofczynski GT, Mundfrom D. Sample sizes when using multiple linear regression for prediction. Educ Psychol Measur 2007;52(5):431-32. doi: 10.1177/0013164407310131.

17. Oliveira Júnior J. Motivos de adesão aos programas de exercícios físicos: um estudo exploratório com praticantes de academias de musculação e ginástica na cidade de Criciúma-SC. 2011. 52 f. TCC (Curso de Educação Física) - Universidade do Extremo Sul Catarinense, Criciúma, 2011.

18. Saba, F. Determinantes da prática de exercícios físicos em academias de ginástica. 1999. Dissertação (Mestrado em Educação Física e Esporte) -Escola de Educação Física e Esporte da USP, São Paulo, 1999.

19. Dishman RK. Handbook of research on sport psychology. New York: MacMillan; 1993. 779 p.

20. Stahl T. The importance of the social enviroment for physically active lifestyle-results from an international study. Social Science and Medice 2001;52:1-10.

21. Mazini Filho ML, Zanell AL, Aidar FJ, Silva AMS, Salgueiro RS, Matos DG. Atividade física e envelhecimento humano: a busca pelo envelhecimento saudável. RBCEH 2010;7(1):97-106.

22. Vale RGS, Dantas EHM. Condicionamento físico e qualidade de vida na academia de ginástica. R Min Educ Fís 2003;11(1):7-24.

23. Spence JC. The effect of exercise on global self-esteem: a quantitative review. J Sport Exerc Psychol 2005;27:311-334.

24. Mazo GZ, Cardoso FL, Aguiar DL. Programa de hidroginástica para idosos: motivação, autoestima e autoimagem. Rev Bras Cineant Desemp Hum 2006;8(2):67-72.

25. Safons MP. Contribuições da atividade física, para a melhoria da autoimagem e autoestima de idosos. Revista Digital Lecturas: Educación Física y Deportes 2000;5(22).

26. Mello MT. Os exercícios físicos e os aspectos psicológicos. Rev Bras Med Esporte 2005;11(3):70-5.

27. Areias MEQ, Guimarães LAM. Gênero e estresse em trabalhadores de uma universidade pública do estado de São Paulo. Psicol Est 2004;9(2):255-262.

28. Carvalho CA. Tratamentos Revolucionários. Boletim da SBGG-RJ 1996;4(10):03.

29. Fox KR. The Physical Self - From Motivation to WellBeing. EUA: Human Kinetics; 1997. 139 p.

30. Vieira VCR, Priere SE, Fisberg M. A atividade física na adolescência. Adolesc. Latino am 2002;3(1):1-14.

31. Coelho-Ravagnanl CF. Efeito de programa para mudança do estilo de vida sobre a percepção de saúde em adultos. Rev Eletr Gest Saúde 2011;2(2):415-26. 